\title{
Distribution of variation over populations
}

\author{
Hans-Rolf Gregorius
}

Received: 24 March 2009/Accepted: 31 March 2009/Published online: 18 April 2009

(c) The Author(s) 2009. This article is published with open access at Springerlink.com

\begin{abstract}
Understanding the significance of the distribution of genetic or phenotypic variation over populations is one of the central concerns of population genetic and ecological research. The import of the research decisively depends on the measures that are applied to assess the amount of variation residing within and between populations. Common approaches can be classified under two perspectives: differentiation and apportionment. While the former focuses on differences (distances) in trait distribution between populations, the latter considers the division of the overall trait variation among populations. Particularly when multiple populations are studied, the apportionment perspective is usually given preference (via $F_{\mathrm{ST}} / G_{\mathrm{ST}}$ indices), even though the other perspective is also relevant. The differences between the two perspectives as well as their joint conceptual basis can be exposed by referring them to the association between trait states and population affiliations. It is demonstrated that the two directions, association of population affiliation with trait state and of trait state with population affiliation, reflect the differentiation and the apportionment perspective, respectively. When combining both perspectives and applying the suggested measure of association, new and efficient methods of analysis result, as is outlined for population genetic processes. In conclusion, the association approach to an analysis of the distribution of trait variation over populations resolves problems that are
\end{abstract}

H.-R. Gregorius ( $\square$ )

Institut für Populations- und ökologische Genetik,

Am Pfingstanger 58, 37075 Göttingen, Germany

e-mail: hgregor@gwdg.de

H.-R. Gregorius

Abteilung Forstgenetik und Forstpflanzenzüchtung,

Büsgenweg 2, 37077 Göttingen, Germany frequently encountered with the apportionment perspective and its commonly applied measures in both population genetics and ecology, suggesting new and more comprehensive methods of analysis that include patterns of differentiation and apportionment.

Keywords Apportionment of variation .

Differentiation between populations .

Diversity within and between populations - Association .

Asymmetry - Fixation - Trait resolution .

Duality of perspectives - Complementarity .

Genetic diversity · Ecological diversity

\section{Introduction}

Particularly in population genetic studies, variation is commonly regarded as being apportioned within and between populations (hereafter referred to as the apportionment perspective). Variation within and variation between populations are accepted to be mutually complementary aspects of the total variation taken across all populations (the population assemblage). This focus accounts for the fact that "organic evolution, in the Darwinian scheme, is a consequence of the conversion of variation among members of an ensemble into differences between ensembles in time and space" (Lewontin 2000, p. 5). More specifically, the focus draws on the expectation that species must have evolved strategies of optimal distribution of their genetic variation over populations with the result that this variation can be maintained and made available for adaptational processes in their populations. At higher levels of biological organization, an analogous argument applies to the distribution of species over communities as an enhancement of ecosystem stability. 
Attempts to quantify the apportionment of variation usually rely on variance and probability arguments. Since the variation distributed over populations is of a qualitative nature (genetic types), variance arguments do not directly apply. This problem is usually bypassed through the use of indicator functions as quantitative substitutes for traits that are actually qualitative. As a consequence, the assessment of variation is limited to simple presence-absence statements. The pertaining indices make use of the basic variance decomposition, in which, the total variance equals the expectation of the conditional variances plus the variance of the conditional expectations, where the conditions are defined by the populations. Wright's $F$ statistics (Wright 1969) for biallelic loci and other related indices (see Weir 1996, for an overview) belong to this category.

The probabilistic analogue to the decomposition of variances is based on probabilities of sampling two objects that differ genetically. The total probability equals the sum of the probabilities of sampling genetically heterogeneous pairs of individuals within the same population and from different populations. This decomposition of probabilities is used in various ways to arrive at indices for "diversity" (variation) within and between populations (among which is Nei's analysis of gene diversity, Nei 1973; for a review see Charlesworth 1998). While there is little disagreement about how to measure diversity within populations, methods to assess diversity between populations are still a matter of inquiry and dispute (see e.g. Manel et al. 2003; Hedrick 2005; Gregorius et al. 2007).

Among the most likely reasons for this situation are conceptual problems of distinguishing the idea of diversity (or variation) between populations (as part of the apportionment perspective) from the idea of differentiation between populations. In population genetics, concepts of differentiation are frequently linked to the measurement of genetic distances between two populations. Distances, however, do not readily fit into the frame of the apportionment perspective, since they cannot, in any sense, be conceived of as measuring variation between populations in terms of the complement of variation within populations. In fact, variation within populations has no direct effect on distances. Even though this was already stressed by Wright (1978, p. 82) in a comparison of his fixation index $F_{\text {ST }}$ with measures of genetic distance and differentiation between populations, the consequences still seem to be rarely recognized, both in conceptual analysis and data analyses.

This situation explains the motivation of the present paper, which is to search for a perspective on the distribution of variation over populations that allows the common perception of diversity (variation) within and between populations to be put into a context that is consistent with the perception of differentiation. Most of the considerations presented for populations apply analogously to communities defined at higher (or lower) levels of biological organization.

\section{The association approach}

Basically, any distribution of variation over populations is characterized by a set of individuals, each of which is identified by two attributes, one of which is defined by the state of a particular trait and the other by population affiliation. In a wide sense, the distribution of the trait variation over populations can therefore be conceived of as a particular association between the trait states and population affiliations of individuals (see Table 2). Thus, if no association exists, the variation of the trait is equally distributed over all populations and, from a probabilistic point of view, stochastic independence is realized between trait state and population affiliation. This situation can be looked at from two different viewpoints. One viewpoint recognizes that "all trait variation resides within populations" (no division of variation among populations) when each individual population represents the total assemblage of populations with respect to the distribution of trait states. The other viewpoint recognizes the same situation as "absence of differentiation for the trait between populations".

The first viewpoint is characteristic of the apportionment perspective according to which the total variation can always be divided into variation that resides within and between populations (see e.g. Rao 1982, p. 29f). In its extremes, the perspective regards all of the variation either to be represented within populations (the total variation is represented in each population, as mentioned above) or to be completely split between populations. The latter case implies the absence of variation within the populations and thus characterizes them as monomorphic or fixed. Consequently, in this case, each trait state is perfectly associated with a particular population. In other words, the trait state of an individual is completely predictable from its population affiliation. This does not exclude perfect association of the same trait state with different populations. Different populations may therefore be fixed for the same trait state, so that they are not completely differentiated for that trait.

The second viewpoint is directed towards recognizing differences or identities in trait distribution between populations. Differences are complete if populations share no trait states, irrespective of the absence or presence of variation within populations. Hence, complete differentiation may occur even though not all of the variation is split between populations. The apportionment perspective thus does not include complete differentiation as one of its extremes but may rather suggest an intermediate assessment of the amount of variation between populations. 
On the other hand, the association approach again arrives at an unambiguous statement in that complete differentiation of populations for a trait can only be realized if each population is perfectly associated with a particular trait state. The population affiliation of an individual is thus completely predictable from its trait state.

It therefore appears that the association approach has the potential to reveal basic relationships between the differentiation and apportionment perspectives. Yet, so far this is only argued for the extremes of the perspectives and may not consistently extend to intermediate situations. More clarity can probably be achieved in this respect by trying to answer the following two questions that refer to basic characteristics of both distributions of trait variation over populations and associations between trait state and population affiliation:

(1) Is there a tendency for individuals holding different trait states to occur in different populations? Can membership in different populations thus to some degree be predicted from difference in trait state?

(2) Is there a tendency of individuals holding the same trait state to reside in the same population? Can identity in trait state thus to some degree be predicted from membership in the same population?

Seeking answers to question (1) will be referred to as the division criterion, while pursuing answers to question (2) may be appropriately termed the concentration criterion. The latter criterion underlies for example the statistic of differentiation considered by Hudson (2000, p. 2011, middle of right column).

The connection of these two criterions to the apportionment and differentiation perspective and to the association approach can be easily established by again starting with the extremes of these criterions. At the one extreme, the absence of tendencies in either of the two criterions clearly implies that populations do not differ in their trait distributions. The two perspectives and the association approach agree in the assessment of this case: all variation resides within populations (no division among populations), no differentiation, no association.

At the other extreme, the tendency in at least one of the criterions is considered to be strict. For the division criterion this implies that membership in the same population prohibits difference in trait state. Hence, all members of a population share the same trait state (populations are monomorphic or fixed), and all variation is split between populations. In other words, the apportionment of trait variation to populations is complete in the sense that the variation is fully divided among populations. Association of trait states with population affiliation thus is perfect, but differentiation need not be complete. For the concentration criterion, strictness of the tendency implies that holding the same trait state only within the same population does not allow members of different populations to share trait states. Consequently, differentiation is complete, association of population affiliation with trait state is perfect, but some of the total variation may reside within populations.

These considerations substantiate the fact that for strict tendencies, the division criterion and the concentration criterion address opposite and asymmetric directions of association between trait state and population affiliation. Besides associations of trait state with population affiliation (called TP-association in the following), the division criterion encompasses the apportionment perspective of variation. On the other hand, the concentration criterion combines the idea of associations of population affiliation with trait state (called PT-association in the following) with the perspective of differentiation of populations for trait variation. Table 1 summarizes the above terms and their correspondences.

Of course, perfect PT-association and thus complete differentiation cannot be realized, if the number of populations exceeds the number of trait states. Along the same line, perfect TP-association and thus complete splitting of the total trait variation between populations (fixation of all populations) cannot occur if there are more trait states than populations. This obvious fact is occasionally overlooked in the analysis of highly polymorphic genetic traits. It will be treated in more detail later in connection with permutation analysis.

To proceed from the characterization of strict tendencies to the characterization of arbitrary tendencies in the division and concentration criterion, it is useful to consider special changes in the distribution of trait states over populations. For example, consider the situation where within-population variation is lowered while maintaining the overall variation. This requires that individuals sharing a trait state be brought together within the same population, which is consistent with the concentration criterion. Trait states that were formerly shared between populations are therefore replaced by states not shared between populations. By this, both the variation and the differentiation between populations are expected to increase. Hence, it is difficult to distinguish the apportionment from the differentiation perspective.

On the other hand, by concentrating like trait states within the same population, predictability of population affiliation from trait state and thus PT-association increases, so that the ambiguity between the apportionment and the differentiation perspective does not carry over to the association approach. An analogous reasoning shows that the ambiguity also arises with the division criterion but can be avoided by turning to TP-associations. Hence, the association approach indeed reveals a basic relationship between the apportionment and the differentiation 
Table 1 Correspondence between perspectives (of the distribution of trait variation over populations), criterions (for the characterization of the perspectives), and associations (between trait state and population affiliation)

\begin{tabular}{lll}
\hline Perspective & Criterion & Association \\
\hline $\begin{array}{l}\text { Apportionment } \\
\begin{array}{l}\text { Division of the overall variation } \\
\text { among populations }\end{array}\end{array}$ & $\begin{array}{c}\text { Division } \\
\text { Individuals holding different trait states } \\
\text { tend to occur in different populations }\end{array}$ & $\begin{array}{c}\text { TP-association } \\
\text { Association of trait state with } \\
\text { population affiliation }^{\mathrm{a}}\end{array}$ \\
$\begin{array}{l}\text { Differentiation } \\
\begin{array}{l}\text { Difference of trait distribution } \\
\text { between populations }\end{array}\end{array}$ & $\begin{array}{c}\text { Concentration } \\
\text { Individuals holding the same trait state } \\
\text { tend to occur in the same population }\end{array}$ & $\begin{array}{c}\text { Ass-association } \\
\text { with trait state }\end{array}$ \\
\hline
\end{tabular}

" Alternatives to the wording "Y is associated with $\mathrm{X}$ " are "Y is predictable from X", "Y depends on X", or "Y is determined by X"

perspective by attributing the former to TP- and the latter to PT-association. The attribution is not limited to the extremes but rather extends to all intermediate situations of the division and concentration criterion. One therefore arrives at the desired result that

- the association approach comprises both the apportionment and the differentiation perspective, where the apportionment perspective is characterized by TP-association and the differentiation perspective by PT-association.

\section{The measurement of association}

A measure taking account of all of the above-mentioned characteristics of association, including those that give rise to the asymmetry in TP- and PT-association, was developed by Gregorius (1998). The measure varies between zero and one, indicating the absence of association and perfect association, respectively. The measure is denoted by $\mathcal{A}(\tau \mid \pi)$ for the association of trait state $(\tau)$ with population affiliation $(\pi)$ (TP-association), and it is denoted by $\mathcal{A}(\pi \mid \tau)$ for the association of population affiliation with trait state (PT-association). Moreover, the relation between PT-association and differentiation argued in the previous section was confirmed by Gregorius (1998), where $\mathcal{A}(\pi \mid \tau)$ was shown to coincide with an established distance-based measure of population differentiation for a specified trait (for further explanations see Appendix; Table 2 provides a simple numerical example for computing associations).

The characterization of the apportionment perspective by TP-association justifies interpretation of $\mathcal{A}(\tau \mid \pi)$ as the degree to which the total trait variation is divided among populations. The two extremes of the absence of division and of complete division are thus specified by $\mathcal{A}(\tau \mid \pi)=0$ and $\mathcal{A}(\tau \mid \pi)=1$, respectively.

In population genetics, the apportionment perspective is frequently referred to by the term fixation. The term is initially borrowed from models of isolated finite populations, in which, over the generations, genetic drift ultimately leads to genetic fixation (monomorphy) of the
Table 2 Association between population affiliation $\pi$ and trait state $\tau$ among 12 individuals distributed over three populations $(a, b, c)$ and showing two trait states $(x, y)$

$$
\begin{aligned}
& \begin{array}{c|cccccccccccc|} 
& 1 & 2 & 3 & 4 & 5 & 6 & 7 & 8 & 9 & 10 & 11 & 12 \\
\hline \pi & \mathrm{a} & \mathrm{a} & \mathrm{a} & \mathrm{b} & \mathrm{b} & \mathrm{b} & \mathrm{b} & \mathrm{b} & \mathrm{c} & \mathrm{c} & \mathrm{c} & \mathrm{c} \\
\tau & \mathrm{x} & \mathrm{x} & \mathrm{x} & \mathrm{x} & \mathrm{x} & \mathrm{y} & \mathrm{y} & \mathrm{y} & \mathrm{x} & \mathrm{x} & \mathrm{y} & \mathrm{y}
\end{array} \\
& \mathcal{A}(\pi \mid \tau)=0.312 \\
& \begin{array}{c|cccccccccccc|} 
& 1 & 2 & 3 & 4 & 5 & 9 & 10 & 6 & 7 & 8 & 11 & 12 \\
\hline \tau & \mathrm{x} & \mathrm{x} & \mathrm{x} & \mathrm{x} & \mathrm{x} & \mathrm{x} & \mathrm{x} & \mathrm{y} & \mathrm{y} & \mathrm{y} & \mathrm{y} & \mathrm{y} \\
\pi & \mathrm{a} & \mathrm{a} & \mathrm{a} & \mathrm{b} & \mathrm{b} & \mathrm{c} & \mathrm{c} & \mathrm{b} & \mathrm{b} & \mathrm{b} & \mathrm{c} & \mathrm{c}
\end{array} \\
& \mathcal{A}(\tau \mid \pi)=0.429
\end{aligned}
$$

joint frequencies of population affiliations and trait states

$$
\begin{gathered}
N(\pi=a, \tau=x)=3, N(\pi=b, \tau=x)=2, N(\pi=c, \tau=x)=2 \\
N(\pi=a, \tau=y)=0, N(\pi=b, \tau=y)=3, N(\pi=c, \tau=y)=2 \\
\text { matrix of joint frequencies } \\
\qquad \begin{array}{c|ccc|}
\tau \downarrow & \mathrm{a} & \mathrm{b} & \mathrm{c} \\
\hline \mathrm{x} & 3 & 2 & 2 \\
\mathrm{y} & 0 & 3 & 2
\end{array}
\end{gathered}
$$

The upper two panels show the same joint distribution of population affiliation and trait state. First panel: individuals ordered for population affiliation (demonstrating association of population affiliation with trait state); second panel: same individuals ordered for trait state (demonstrating association of trait state with population affiliation)

populations. This is in accordance with perfect TP-association. However, it neglects the complementarity of variation within and between populations, by which the absence of variation within populations implies that all variation is divided among populations. Moreover, usage of the term fixation may become vague when it is extended to situations where populations are "more or less fixed". In fact, while the absence of variation between populations has intuitive appeal, it is difficult to conceive of situations where fixation is completely or even partially absent.

The complementarity principle inherent in the apportionment perspective operates within the limits set by the 
total trait variation present in the assemblage of all populations. It therefore seems logical to consider the amount of variation between populations to correspond to the difference between the total variation and the (average) variation within populations. This is particularly tempting, since the quantification of both total and within-population variation is based on the same computational rules. In fact, most approaches to the measurement of variation between populations follow this logic. Yet, as pointed out by Rao (1982), the difference between the measures of variation within and between populations becomes meaningless if these measures do not show certain concavity properties (in population mixtures). These properties are mandatory for maintenance of the complementarity principle. If they are not realized, it may happen that the "variation within populations" exceeds the "total variation" (implying negative "variation between populations"). It is, however, difficult to identify the basic biological or conceptual principles that force measures of variation to be concave.

By turning to associations, conceptual problems of this kind disappear. To see this, recall that the division criterion was shown to characterize the apportionment perspective by TP-association. In particular, by increasing the tendency of individuals with different trait states to occur in different populations, the variation between populations increases. This can of course only be realized by reducing the variation within populations, which confirms the idea that variation within and between populations ought to be complementary. For this reason, $\mathcal{A}(\tau \mid \pi)$ is a consistent measure of the apportionment of trait variation to populations in that it specifies the degree to which the variation is divided among populations. By default, the amount of variation not divided among populations refers to variation within populations.

Later on in this paper, relations between the association measures $\mathcal{A}$ and $F_{\mathrm{ST}}$ will be considered in more detail. In population genetics, $F_{\mathrm{ST}}$ or its extension $G_{\mathrm{ST}}$ to multiple alleles is alternately addressed as a measure of population differentiation, population fixation, and apportionment of genetic variation to populations. For the moment it may suffice to note that, not surprisingly, $F_{\mathrm{ST}}\left(G_{\mathrm{ST}}\right)$ has been criticized for its failure to appropriately reflect the one or the other of these readings (for more recent criticisms see e.g. Hedrick 2005, or Gregorius et al. 2007, p. 199). Nevertheless, this has not diminished the popularity of the index.

\section{Apportionment and differentiation are dual perspectives}

The difference in interpretation of the two directions of association is solely due to our bias towards looking at the way in which trait variation is distributed within and between populations. In fact, one could just as well consider the way in which population affiliation of individuals is distributed over individual trait states, in which case perfect association of population affiliation with trait state (i.e. $\mathcal{A}(\pi \mid \tau)=1$ ), for example, could be conceived of as fixation to a single population among the carriers of a specific trait state (only one population contributes to a trait state). This is of course equivalent to the statement of complete differentiation between populations for trait states. Hence, PT-association can be looked at from both the apportionment and the differentiation perspective, depending on the preferred representation of the joint distribution of trait states and population affiliations (trait states distributed over populations, or population affiliations distributed over trait states).

Conversely, differentiation among carriers of individual trait states for population affiliation is complete, if trait states are perfectly associated with population affiliation (i.e. $\mathcal{A}(\tau \mid \pi)=1$, only one trait state is represented in each population). This is equivalent to monomorphy of all populations, so that TP-association can also be looked at from the differentiation perspective and not only from the apportionment perspective. One thus arrives at the interesting observation that the two perspectives can be transformed (converted) into each other by simply inverting the order of association between trait states and population affiliation. The various relationships between representation of the joint distribution, the differentiation and apportionment perspectives, and direction of association are listed in Table 3.

Table 3 The differentiation and apportionment perspectives with their pertaining directions of association between trait states and population affiliation for the two representations of the joint distribution of trait states and population affiliations

\begin{tabular}{llll}
\hline Representation of distribution & Perspective $\longrightarrow$ & Direction of association $\longrightarrow$ & Measure \\
\hline Trait states distributed over populations & Differentiation & PT-association & $\mathcal{A}(\pi \mid \tau)$ \\
\cline { 2 - 4 } & Apportionment & TP-association & $\mathcal{A}(\tau \mid \pi)$ \\
\hline Population affiliation distributed over trait states & Differentiation & TP-association & $\mathcal{A}(\tau \mid \pi)$ \\
\cline { 2 - 4 } & Apportionment & PT-association & $\mathcal{A}(\pi \mid \tau)$ \\
\hline
\end{tabular}


It is therefore justified to state duality between the apportionment and the differentiation perspectives, where the duality is established by inverting the direction of association within each of the two representations of the joint distribution, i.e. by swapping $\pi$ and $\tau$ in $\mathcal{A}$ (see Table 3 ). In other words, for the distribution of trait states over populations, $\mathcal{A}(\pi \mid \tau)$ measures the differentiation between populations for trait variation, while $\mathcal{A}(\tau \mid \pi)$ measures the differentiation between trait states for population affiliation. Analogously, for the distribution of population affiliation over trait states, $\mathcal{A}(\tau \mid \pi)$ measures the division of trait variation among populations, while $\mathcal{A}(\pi \mid \tau)$ measures the division of population affiliation among trait states. This implies that the duality exists at two levels depending on the representation of the joint distribution: (1) opposite directions of association can be conceived to both measure differentiation or both measure apportionment, (2) the same direction of association can be conceived as measuring differentiation and apportionment (see Table 3).

The greatest advantage of the duality probably lies in the fact that, whatever perspective is considered to be more intuitively appealing or closer to the problem, it can be applied to understand both PT- and TP-association. Thus, if for any reason, the differentiation perspective is preferable to the apportionment perspective, PT-association would be interpreted in terms of the distribution of trait states over populations, while TP-association would be understood through the distribution of population affiliation over trait states (the arrangements in Table 2 follow this perspective). Any of the other combinations that can be taken from Table 3 may serve its special purpose. The following section provides an example of how these combinations can be used in the interpretation of population genetic processes.

\section{Population genetic interpretation of associations}

The significance of association measures for the analysis of biological processes becomes more apparent when recalling the ultimate objective of measuring association, namely the detection of cause-effect relationships. Such relationships between two variables become more likely, the more strong variable is associated with (and thus depends on, is determined by, or is predictable from) the other. The dependence or determination need not be functional and may be mutual, including asymmetric effects of additional variables on the two under observation. Hence, if the association of population affiliation with a genetic trait exceeds the association in the opposite direction (i.e. $\mathcal{A}(\pi \mid \tau)>\mathcal{A}(\tau \mid \pi))$, then the population affiliation of individuals depends more strongly on their genetic characteristics than vice versa.

At first sight, statements of this kind may appear obscure. They gain clarity when considering the interpretations of the measure $\mathcal{A}$ in terms of differentiation and apportionment together with the implied distributional characteristics. Particularly, large associations $\mathcal{A}(\pi \mid \tau)$ result from situations where carriers of a particular genotype tend to occur within the same population. Populations would be well differentiated in this case. The possibility that in addition they are distinctly polymorphic would in turn lower the predictability of an individual's genotype from its population affiliation, so that TP-association and thus $\mathcal{A}(\tau \mid \pi)$ is deflated. In this case, population affiliation is obviously more strongly determined by trait state than vice versa.

However, in most cases, the simple ordering of the two associations is not likely to provide the information required to clearly distinguish between the effects of different population genetic processes. Such information is rather expected from combinations of distinct differences between the associations. Table 4 provides a listing of the relevant combinations of large and small values for the PTand TP-associations together with their implied distribution characteristics. As will be elaborated in the next section, the wordings "large" and "small" have to be understood within the frame set by the overall number and frequencies of trait states and by the number of populations and their sizes. They are therefore referred to as "relatively large" and "relatively small" in Table 4.

To simplify the argument on the potential forces that bring about the four cases in Table 4, it is useful to distinguish homogenizing from diversifying forces acting between and within populations. Among the diversifying forces acting between populations are random drift, differential selection due to adaptive variation of environmental factors, and non-recurrent mutation. Between

Table 4 Effects of associations between population affiliation and genetic traits on the distribution of genetic variation over populations

(1) $\uparrow \mathcal{A}(\pi \mid \tau), \downarrow \mathcal{A}(\tau \mid \pi)$ : populations tend to be genetically differentiated and polymorphic

(2) $\downarrow \mathcal{A}(\pi \mid \tau), \uparrow \mathcal{A}(\tau \mid \pi)$ : populations show both reduced genetic differentiation and polymorphism; this implies low genetic polymorphism in the population assemblage

(3) $\uparrow \mathcal{A}(\pi \mid \tau), \uparrow \mathcal{A}(\tau \mid \pi)$ : populations are genetically well differentiated and show low polymorphism

(4) $\downarrow \mathcal{A}(\pi \mid \tau), \downarrow \mathcal{A}(\tau \mid \pi)$ : populations show little genetic differentiation irrespective of their degrees of polymorphism

$\uparrow$ and $\downarrow$ refer to "relatively large" and "relatively small", respectively 
populations, homogenizing forces are exerted by migration and uniformly acting selection (all populations subjected to similar selection regimes). In addition, lack of differentiation (between populations) may be due to joint descent in the absence of diversifying forces.

Within populations, homogenization is understood to act towards reduction of polymorphism as is expected from directional selection or (random) drift in small and reproductively sufficiently isolated populations. Diversification within populations may result from adaptation to heterogeneous habitats, pronounced gene flow (reproductive connectedness) among genetically differentiated populations, and non-recurrent mutation.

The four potential observations stated in Table 4 can thus be explained as follows:

Case (1) Populations are maintained genetically different and polymorphic by non-recurrent mutation, by adaptation to habitats which are heterogeneous within and between populations, and by balancing selection (overdominance, negative frequency dependence) acting differentially between populations. The lower the gene-flow between populations, the more genetically differentiated they can become.

Case (2) Low genetic variation within populations combined with low genetic differentiation is most likely the result of common descent from an ancestral population that recently experienced a bottleneck.

Case (3) Genetic drift in small and reproductively sufficiently isolated populations can lead to this situation.

Case (4) This situation is most likely to result from strong gene flow among the populations particularly in the presence of high polymorphism. Intermediate or low polymorphism may indicate uniformly acting selection possibly combined with recurrent mutation.

Clearly, these explanations first have the status of hypotheses suggested by observations of associations between trait state and population affiliation. Since the measures of association clearly distinguish the four categories of hypotheses, proofs as to the significance of the observed category concentrate on testing for the possibility of randomness of the observation. Moreover, in analytic or simulation analyses of models, the behavior of associations can be studied under various types of selection, mutation, migration, or drift as well as combinations of these acting on population systems (such as metapopulations). This may reveal association characteristics (in terms of differentiation/apportionment) of population genetic processes that previously escaped notice.

\section{Analysis of associations}

Any particular distribution of trait variation over populations can be realized at random, and this transfers to the pertinent associations. It is therefore desirable to distinguish amounts of association that can typically be produced by purely randomly acting forces from those that are due to non-random forces. This requires consideration of frequency distributions of measures of association as they result from randomness. Within such distributions, the position of the observed association value can be assessed by the frequency of situations (or system states) that yield larger values than those observed and by the frequency of situations yielding smaller values. In many if not most situations, randomness can be effectively tested with the help of (conditional) permutation analyses, in which all permutations of one variable (trait state, say) over the other variable (population affiliation) are considered.

For simplicity, consider first the differentiation perspective with its measure $\mathcal{A}(\pi \mid \tau)$ of PT-association. Small frequencies of permutations that exceed the observed differentiation then indicate that the observation is larger than can be expected under purely randomly acting forces. Therefore, diversifying forces in the form of differential selection or non-recurrent mutation [part of case (1) in the previous section] can be inferred to clearly override randomly acting and homogenizing forces. Non-recurrent mutation is likely to produce high degrees of genetic diversity (within and between populations), and by this it may be distinguished from differential selection.

Conversely, if the frequency of permutations that fall below the observed differentiation is small, homogenizing forces can be inferred to be dominant. In this case, further distinction between migration and uniformly acting selection [part of case (4) in the previous section] may be difficult and may require additional information such as on the type of genetic marker. Generally, however, one expects migration to be a homogenizing force that affects many loci, while uniformly acting selection affects only a comparatively small number of loci. Depending on the time elapsed, joint descent can also be held responsible for significant lack of differentiation. In particular, it is unlikely that large parts of the genome remain the same between more or less isolated populations over several generations. This may help to distinguish joint descent from gene flow, since the latter is more likely to produce low differentiation for large numbers of loci.

To obtain the full information available from an analysis of associations, it is necessary to consider both the differentiation and the apportionment perspective together with their measures $\mathcal{A}(\pi \mid \tau)$ and $\mathcal{A}(\tau \mid \pi)$. The relevant combinations and relations between associations in terms or "large" and "small" are listed in Table 4. However, recall 
that in (conditional) permutation analyses, the observed associations are considered large, for example, solely because they are rarely exceeded among all permutations. This does not imply that the observed association ought to be close to 1 . The reasons may be twofold in that the marginal constraints (specified by the number and frequencies of trait states as well as by the number of populations and their sizes) allow realization of only a restricted range of associations, or in that it is unlikely under these constraints to find associations exceeding or falling below a given threshold. Any assessment of the "size" of an observed association can therefore be carried out relative to the marginal constraints or, in addition, with reference to a significance level.

Both kinds of assessment, by marginal constraints and by significance level, are addressed in Table 4 by the wording "relatively large" and "relatively small". In a permutation analysis of the population genetic hypotheses pertaining to the individual cases in Table 4, the assessment will primarily take place on the basis of significance levels. It may thus readily happen that the observed values of $\mathcal{A}(\pi \mid \tau)$ and $\mathcal{A}(\tau \mid \pi)$ are intermediate in absolute value but are "relatively" large and small, respectively, with reference to the marginal constraints or to the level of significance.

Marginal constraints may be quite common. As was noted earlier, TP-association cannot be perfect if the number of trait states exceeds the number of populations. Similarly, perfect PT-association is ruled out if there are more populations than trait states. The frequencies of trait states and population sizes are further sources of constraints. Even when the number of populations equals the number of trait states, perfect TP- or PT-association can be realized only if to each population there corresponds a trait state such that the size of that population equals the number of individuals holding that trait state.

If more subtle ranking relationships between PT- and TP-association rather than situations of extreme divergence between the two are to be analyzed, consideration of the difference $\mathcal{A}(\pi \mid \tau)-\mathcal{A}(\tau \mid \pi)$ suggests itself. An observed positive difference would then be excluded as resulting from pure random effects if it is rarely exceeded among all permutations. Special care, however, must be taken in cases where, for example, an observed negative difference is rarely exceeded among the permutations. This may be due to forms of the marginal constraints that allow almost only for negative differences. Such cases, but also less extreme ones, may give rise to further analysis of the forces that can bring about the respective constraints. Appropriate methods of analysis will then have to resort to the modeling of these constraints and provide suitable estimates and tests of associations.
Testing of such models usually requires consideration of populations as samples from a hypothetically infinite universe of populations, where the associations in the universe are to be estimated from the sample of populations. Since there is no limit to the number of populations in the universe, while the number of trait states is usually finite, perfect $\mathrm{PT}$-associations are ruled out. For TP-associations, however, this restriction does not exist, and trait variation may well be fully divided among populations with the result of fixation in all populations.

\section{Concluding remarks}

Among the most frequently applied indices of genetic variation in the analysis of population genetic models and data is $F_{\mathrm{ST}}$ (or $G_{\mathrm{ST}}$ ). A comparison with the present association measure $\mathcal{A}$ and its perspectives is thus mandatory. The various verbal descriptions of $F_{\mathrm{ST}}$ together with its formal representation characterize this index as the difference between the total diversity $\left(D_{\mathrm{T}}\right.$, say) and the average diversity within populations $\left(D_{\mathrm{W}}\right.$, say) divided by the total diversity, i.e. $F_{\mathrm{ST}}=\left(D_{\mathrm{T}}-D_{\mathrm{W}}\right) / D_{\mathrm{T}}$. In essence, diversity is measured by the probability of sampling different genetic variants (see e.g. Nei, 1973, where $D_{\mathrm{T}}, D_{\mathrm{W}}$, $D_{\mathrm{T}}-D_{\mathrm{W}}$ and $F_{\mathrm{ST}}$ correspond to $H_{\mathrm{T}}, H_{S}, D_{\mathrm{ST}}$ and $G_{\mathrm{ST}}$ ). The same two diversities are used in ecology (where $D_{\mathrm{W}}$ and $D_{\mathrm{T}}$ are referred to as $\alpha$ - and $\gamma$-diversity) to measure $\beta$ diversity as the gain in species diversity due to differences between species collections or communities. The complementarity principle again provides the basis for $\beta$-diversity, which is usually expressed as $D_{\mathrm{T}} / D_{\mathrm{W}},\left(D_{\mathrm{T}}-D_{\mathrm{W}}\right) / D_{\mathrm{W}}$ or $D_{\mathrm{T}}-D_{\mathrm{W}}$ (in ecology different measures of diversity are used; for the Shannon index, usually $\beta=D_{\mathrm{T}}-D_{\mathrm{W}}$ is used; see e.g. Vellend 2001).

Obviously, the idea that the total variation can be partitioned into variation within and between collections is common to all of these indices. They therefore belong to the apportionment perspective. Indeed, all of the indices assume their maximum values for given total variation only if all collections are fixed for a single trait state (genetic variant, species, etc.), i.e. if the total variation is fully divided among collections. Neither $F_{\mathrm{ST}}$ nor its $\beta$ analogues can be classified under the differentiation perspective. The concern of Charlesworth et al. (1997) that $F_{\mathrm{ST}}$ depends "on both genetic divergence and genetic diversity within populations", and that it therefore does not sufficiently distinguish between processes that imply reduction of genetic diversity within and genetic divergence between populations (p. 167), can probably be attributed to not clearly separating the apportionment from the differentiation perspective. The explanations provided in the above section on 
"population genetic interpretation of associations" may be useful in resolving some of the authors's concerns.

Consideration of this kind appear to have little relevance in ecology, since there the differentiation perspective does not seem to have received much, if any, attention (which may be considered a serious omission). In population genetics, however, it is essential, and its neglect has lead to the confusion mentioned in the introduction and to the concerns expressed in the above-cited paper of Charlesworth et al. (1997). Over a number of distribution scenarios simulated when preparing the present paper, a sizable (but far from complete) positive correlation was found between $F_{\text {ST }}$ and $\mathcal{A}(\tau \mid \pi)$, while a distinctly lower positive correlation was observed between $F_{\mathrm{ST}}$ and $\mathcal{A}(\pi \mid \tau)$.

Another characteristic of the above-mentioned common indices of apportionment is to be found in their frequent reference to the term "diversity" rather than "variation". It has been repeatedly called to attention that, in its proper sense, the term diversity is intrinsically aimed at describing an equivalent of the effective number of distinguishable types observable in a collection of objects. Most of the common indices of diversity meet this condition or can be transformed to do so (for a more recent reminder see e.g. Jost 2006, or Gregorius and Gillet 2008). These indices, although they are aimed at measuring variation within populations, are used in the design of all of the $F_{\mathrm{ST}}$ and $\beta$ indices of apportionment (Shannon's measure of information may require a special classification). The indices can therefore be classified as diversity-based measures of apportionment. The implied condition that, for purely formal reasons, the diversity indices must be concave does not explicitly enter into the biological or conceptual argumentation of these measures of apportionment. Moreover, it has to be taken into consideration that the diversity of individual populations may exceed the total diversity even for concave indices.

In fact, measures of differentiation and apportionment make statements relative to the trait variation present in the total population assemblage. Hence, if they are to be supplemented by statements on the variability of the individual populations or of the total population assemblage, this can and probably should be based on separately argued diversity indices. A closer look at the literature reveals that in the vast majority of studies, $F_{\mathrm{ST}}$ values are indeed communicated together with diversity indices (such as "allelic richness") that are not part of the design of $F_{\mathrm{ST}}$. The same holds true for measures of $\beta$ "diversity". Apparently, no need is felt to make the measurement of apportionment of variation depend on the measurement of its diversity.

The practical consequences of confusing the differentiation, apportionment and diversity perspectives are far too numerous to be listed in this paper. It may therefore suffice to briefly address just one aspect of more basic significance: the dependence of the results of analyses on the chosen or available degree of resolution. The term resolution is understood here to apply to both the distinguishability of individuals for their trait state and for their population affiliation. The variants obtainable by electrophoretic separation of a particular isozyme, for example, may be further resolved by sequencing of the enzyme molecules and even more so by sequencing of the coding DNA strands. In the same way, by inclusion of additional information on habitat characteristics, subpopulation structure may become visible that formerly escaped observation.

Higher trait resolution will increase (not always strictly) the differentiation between populations. The measure $\mathcal{A}(\pi \mid \tau)$ is known to fulfill this condition strictly. In contrast, from the apportionment perspective, enhanced trait resolution may (but need not) have the opposite effect. This is easily recognized from inspection of the case of full division of trait variation among populations. An increase in trait resolution may then turn formerly monomorphic into polymorphic populations and by this will decrease both $\mathcal{A}(\tau \mid \pi)$ and $F_{\mathrm{ST}}$ (for $F_{\mathrm{ST}}$ it was demonstrated that this can also happen for formerly polymorphic populations, see the Appendix in Gregorius et al. 2007). Conversely, if additional subpopulation structure is revealed, then the total variation is divided among more populations, so that $\mathcal{A}(\tau \mid \pi)$ increases (not always strictly; the proof that $\mathcal{A}(\tau \mid \pi)$ fulfills this condition is the same as for $\mathcal{A}(\pi \mid \tau)$ considering the duality property). Both kinds of resolution effects are thus consistently mapped by associations but may remain undetected by focussing on $F_{\mathrm{ST}}$ analyses.

Beyond this, the association measures $\mathcal{A}$ offer opportunities for the analysis of patterns of differentiation and apportionment. It was shown by Gregorius (1998), that the average association $\mathcal{A}(\pi=a \mid \tau)$ of a particular population $a$ with the trait states measures the distance in trait distribution between that population and the totality of the remaining populations (also see Appendix). Hence, $\mathcal{A}(\pi=$ $a \mid \tau)$ quantifies the contribution of population $a$ to the overall differentiation between populations. The resulting pattern of distances can be used to treat various problems ranging from the identification of centers of dispersion (populations with lowest distances) to the identification of variable degrees of isolation of individual populations. By virtue of the duality property, the quantities $\mathcal{A}(\tau=b \mid \pi)$ analogously quantify the contribution of trait state $b$ to the division of the total trait variation among populations. By this, trait states can be ranked according to the degree to which they occur in populations in which other trait states do not occur (where they dominate all other trait states). Individuals with trait state $b$ and showing superior adaptation to conditions realized in particular populations can thus be expected to account for large $\mathcal{A}(\tau=b \mid \pi)$ values. 
Summarizing the above considerations and demonstrations, we can conclude that the commonly used apportionment perspective of the distribution of variation over populations should generally be supplemented by the differentiation perspective in the analysis of distributions of trait variation over populations. The proposed measure of association is suitable for the implementation of the analysis, since it covers both perspectives on a common conceptual basis and provides the means for an assessment of patterns of differentiation and apportionment. The measure also avoids the conceptual problems inherent in the common diversity-based indices of apportionment.

Acknowledgments The comments of two anonymous reviewers are appreciated especially for helping to improve the representation of some of the concepts as well as the writing.

Open Access This article is distributed under the terms of the Creative Commons Attribution Noncommercial License which permits any noncommercial use, distribution, and reproduction in any medium, provided the original author(s) and source are credited.

\section{Appendix}

The measurement of association rests on the basic idea that membership of a particular population $\pi=a$, say, is positively associated with holding a particular trait state $\tau=x$, say, if $x$ is more frequent in population $a$ than in the population assemblage, i.e. if $P(\tau=x \mid \pi=a)>P(\tau=x)$. The latter is equivalent to $P(\tau=x \mid \pi \neq a)<P(\tau=x)$, which puts into focus the deficit of $x$-individuals outside of population $a$. Analogously, $\pi=a$ is negatively associated with $\tau=x$ if $P(\tau=x \mid \pi=a)<P(\tau=x)$. A natural measure $\mathcal{A}(\pi=a \mid \tau=x)$ of association of $\pi=a$ with $\tau=x$ is therefore provided by the proportion by which the frequency of individuals holding trait state $x$ in the population assemblage is reduced either among the members of population $a$ or among individuals not belonging to population $a$, i.e.

$$
\begin{aligned}
\mathcal{A}(\pi & =a \mid \tau=x) \\
& =\frac{P(\tau=x)-\min \{P(\tau=x \mid \pi=a), P(\tau=x \mid \pi \neq a)\}}{P(\tau=x)}
\end{aligned}
$$

Obviously, $0 \leq \mathcal{A}(\pi=a \mid \tau=x) \leq 1, \mathcal{A}(\pi=a \mid \tau=x)=1$ if all individuals holding trait state $x$ either belong to population $a$ (positive association) or do not belong to population $a$ (negative association). $\mathcal{A}(\pi=a \mid \tau=x)=0$ exactly if $x$-individuals are equally represented within and outside of population $a$ (stochastic independence). The overall association of population affiliation with trait state then results as the average of the individual associations, i.e. (see Gregorius 1998)

$$
\begin{aligned}
\mathcal{A}(\pi \mid \tau)= & \sum_{a, x} \mathcal{A}(\pi=a \mid \tau=x) \cdot P(\pi=a) \cdot P(\tau=x) \\
= & \sum_{a} P(\pi=a) \cdot \frac{1}{2} \sum_{x} \mid P(\tau=x \mid \pi=a) \\
& -P(\tau=x \mid \pi \neq a) \mid
\end{aligned}
$$

$\mathcal{A}(\pi \mid \tau)$ thus turns out as the average difference (distance) in trait distribution between populations and their respective complements. This equals the index $\delta$ suggested by Gregorius and Roberds (1986) for the measurement of genetic differentiation between subpopulations.

The inverse associations of trait state with population affiliation, i.e. $\mathcal{A}(\tau=x \mid \pi=a)$ and $\mathcal{A}(\tau \mid \pi)$, can be obtained by simply exchanging the order of population affiliation and trait state in all of the above demonstrations. In particular, $\mathcal{A}(\tau \mid \pi)$ measures the average difference in the distribution of population membership between trait states and their respective complements.

\section{References}

Charlesworth B (1998) Measures of divergence between populations and the effect of forces that reduce variability. Mol Biol Evol 15:538-543

Charlesworth B, Nordborg M, Charlesworth D (1997) The effects of local selection, balanced polymorphism and background selection on equilibrium patterns of genetic diversity in subdivided inbreeding and outcrossing populations. Genet Res 70:155-174

Gregorius H-R (1998) Measuring association between two traits. Acta Biotheoretica 46:89-98

Gregorius H-R, Degen B, König A (2007) Problems in the analysis of genetic differentiation among populations-a case study in Quercus robur. Silvae Genetica 56:190-199

Gregorius H-R, Gillet EM (2008) Generalized Simpson-diversity. Ecol Model 211:90-96

Gregorius H-R, Roberds JH (1986) Measurement of genetical differentiation among subpopulations. Theor Appl Genet 71: $826-834$

Hedrick PW (2005) A standardized genetic differentiation measure. Evolution 59:1633-1638

Hudson RR (2000) A new statistic for detecting genetic differentiation. Genetics 155:2011-2014

Jost L (2006) Entropy and diversity. OIKOS 113:363-375

Lewontin R (2000) The problems of population genetics. In: Singh \& Krimbas, pp 5-23

Manel S, Schwartz MK, Luikart G, Taberlet P (2003) Landscape genetics: combining landscape ecology and population genetics. Trend Ecol Evol 18:189-197

Nei M (1973) Analysis of gene diversity in subdivided populations. Proc Natl Acad Sci USA 70:3321-3323

Rao CR (1982) Diversity and dissimilarity coefficients: A unified approach. Theor Popul Biol 21:24-43

Singh RS, Krimbas CB (eds) (2000) Evolutionary genetics: from molecules to morphology. Cambridge University Press, London

Vellend M (2001) Do commonly used indices of $\beta$-diversity measure species turnover? J Veget Sci 12:545-552

Weir BS (1996) Genetic data analysis II. Sinauer Associates, Sunderland 
Wright S (1969) Evolution and the genetics of populations, vol 2. The theory of gene frequencies. The University of Chicago Press, Chicago
Wright S (1978) Evolution and the genetics of populations, vol 4. Variability within and among natural populations. The University of Chicago Press, Chicago 